\title{
A Comparative Study of Simulated Annealing with Different Cooling Schedules for Geometric Optimization of a Heat Transfer Problem According to Constructal Design
}

\author{
Um Estudo Comparativo do Simulated Annealing com Diferentes Cooling Schedules para a \\ Otimização Geométrica de um Problema de Transferência de Calor de Acordo com o \\ Constructal Design
}

\author{
G. V. Gonzales ${ }^{1, *} ;$ L. R. Emmendorfer'; L. A. Isoldi'; E. da S. D. Estrada ${ }^{2}$; L. A. \\ O. Rocha ${ }^{2}$, E. D. dos Santos ${ }^{1}$ \\ ${ }^{1}$ Programa de Pós-Graduação em Modelagem Computacional (PPGMC), Universidade Federal do Rio Grande \\ (FURG),96021-900, Rio Grande, Brasil \\ ${ }^{2}$ Department of Mechanical Engineering (DEMEC), Universidade Federal do Rio Grande do Sul (UFRGS),90050- \\ 170, Porto Alegre, Brasil \\ *gillvelleda@gmail.com
}

(Recebido em 22 de setembro de 2014; aceito em 29 de dezembro de 2014)

The Simulated Annealing is a widely used meta-heuristic to combinatorial and optimization problems studied since 1983, when it was first proposed. This algorithm has a particular parameter, the cooling schedule (specifically the decrease of temperature) which is still defined for each problem empirically. This study has the aim to find the best cooling schedule(s) for geometric optimization of a heat transfer problem. More specifically it is performed the geometrical evaluation of an isothermal Y-shaped cavity intruded into conducting solid wall with internal heat generation. In this research, Constructal Design is employed to determine the constraints and objectives for geometric evaluation of this problem. The cooling schedule(s) recommended here will contribute for future applications of Simulated Annealing heuristic in association with Constructal Design to seek for the optimal shapes of complex geometries in heat transfer problems, as well as, evaluate the influence of geometric parameters over thermal performance of the problem. Six different methods of decrease of temperature are evaluated in twenty samples of thirty simulations for each cooling schedule. From these simulations, it was extracted the percentage of convergence to the global optimal point. Results showed that the Fast cooling schedule led to the worst performance in the seek for the global optimal shapes, while the three hybrid cooling schedules proposed here are the most recommended to find the optimal geometrical configurations in heat transfer problems in association with Constructal Design (BoltzExp, ConstExp1 and ConstExp2).

Keywords: Constructal Design, Simulated Annealing, Geometric Optimization

O Simulated Annealing é uma meta-heurística amplamente utilizada para problemas combinatórios e de otimização estudada desde 1983, quando foi proposta. Este algoritmo possui um parâmetro particular, o cooling schedule (especificamente o decaimento de sua temperatura) que ainda é definido de forma empírica para cada tipo de problema. Este estudo tem como objetivo encontrar o melhor cooling schedules(s) para a otimização geométrica de um problema de transferência de calor. Mais especificamente, é realizada a avaliação geométrica de uma cavidade isotérmica em forma de Y inserida em um sólido com geração de calor interna. Nesta pesquisa, o método Constructal Design é empregado na determinação de restrições e objetivos para a avaliação geométrica deste problema. Os cooling schedules recomendados aqui, contribuirão para futuras aplicações da heurística Simulated Annealing em associação ao Constructal Design na busca pelas formas ótimas de geometrias complexas em problemas de transferência de calor, assim como, na avaliação da influência dos parâmetros geométricos sobre o desempenho térmico do problema. Seis diferentes métodos de decaimento de temperatura são avaliados em vinte amostras de trinta simulações para cada cooling schedule. A partir destas simulações, foi extraída a porcentagem de convergência ao ponto de ótimo global. Os resultados mostram que o cooling schedule Fast obteve a pior performance na busca pelas geometrias ótimas globais, enquanto que três cooling schedules híbridos propostos aqui são os mais recomendados na busca pelas configurações geométricas ótimas em problemas de transferência de calor em associação ao Constructal Design (BoltzExp, ConstExp1 e ConstExp2).

Palavras-chave: Constructal Design, Simulated Annealing, Otimização Geométrica 


\section{INTRODUCTION}

The algorithm Simulated Annealing (SA) is a meta-heuristic proposed by Kirkpatrick [1]. It is an implementation of Metropolis et al. [2] method in a sequence of iteration controlled by a temperature parameter, based on physic annealing process. According to Kirkpatrick [1] the temperature parameter and the behavior of temperature decay is defined empirically. This parameter is responsible for the control of temperature in the algorithm and is called cooling schedule. This parameter has the highest influence over the search for the optimal results $[3,4]$. There are in literature several studies about the best cooling schedules or variants of classical SA, see Refs. [5-13]. However, there is no universally recommended cooling schedule to seek for the best geometries in heat transfer problems.

Constructal Design derives from Constructal Theory, which is the view that geometry patterns of any animate or inanimate flux systems, which are seen in nature is not result of chance, but emerged by a physical principle, the Constructal Law [14,15]. Constructal Law states that "For a finite-size flow system to persist in time (to live), its configuration must evolve in such a way that provides easier access to the currents that flow through it". The form to employ Constructal Law for geometric evaluation of flux systems is the Constructal Design [14], which is a method based on objectives and restrictions used to minimize the imperfections of the investigated systems. This method has also been applied for improvement of performance in engineering problems. Recently, several C, T, Y-shaped cavities, as well as, complex and multiple cavities has been studied by means of Constructal Design, see Refs. [16-24].

The problem studied here is a Y-shaped cavity that is intruded into a conducting solid wall with internal heat generation. The heat is just only removed by Y-shaped cavity and the objective is to optimize its geometry to minimize the thermal resistance of the solid. In general, as more complex is the cavity geometry to be evaluated more degrees of freedom (DOF) are required to define. The optimization of this kind of problem requires a large computational effort due to the high number of required simulations for complete optimization. In this framework, recent studies [21, 22] employed genetic algorithms (GA) to seek for the best shapes. The achieved results for few degrees of freedom were compared with those achieved with Exhaustive Search (ES) which consists on the simulation of all geometric possibilities. Afterwards, new results were proposed using only GA for the whole degrees of freedom of the problem.

The employment of Simulated Annealing (SA) in association with Constructal Design for geometrical optimization of cavities has not been studied in literature at the authors knowledge, representing a novel important study. Then, the study of appropriate annealing cooling schedule is also an important subject. The application of algorithm without adjusted configurations can lead to errors in the achievement of global optimal shapes, as well as, in the evaluation of influence of each geometric parameter (DOF) over thermal performance of the problem, mainly in cases where it is not possible to compare the results with those achieved with ES. In this sense, this study intends to reach to a recommendation about the best cooling schedules for a reliable optimization with SA meta-heuristic for future optimization of complex cavities into Constructal Design framework.

\section{MATHEMATICAL MODELING}

Figure 1 shows the computational domain of the studied problem. The Y-shaped cavity acts removing the heat generated by solid domain (gray region). The seek for the optimal geometry of the cavity is given by variation of the degrees of freedom: $H / L, t_{1} / t_{0}, L_{1} / L_{0}$ and $\alpha$. The temperature field inside the solid domain is solved for every studied cases and the maximum temperature is collected. The best thermal performance of the system is obtained when the maximum temperature inside the solid domain is minimized. 


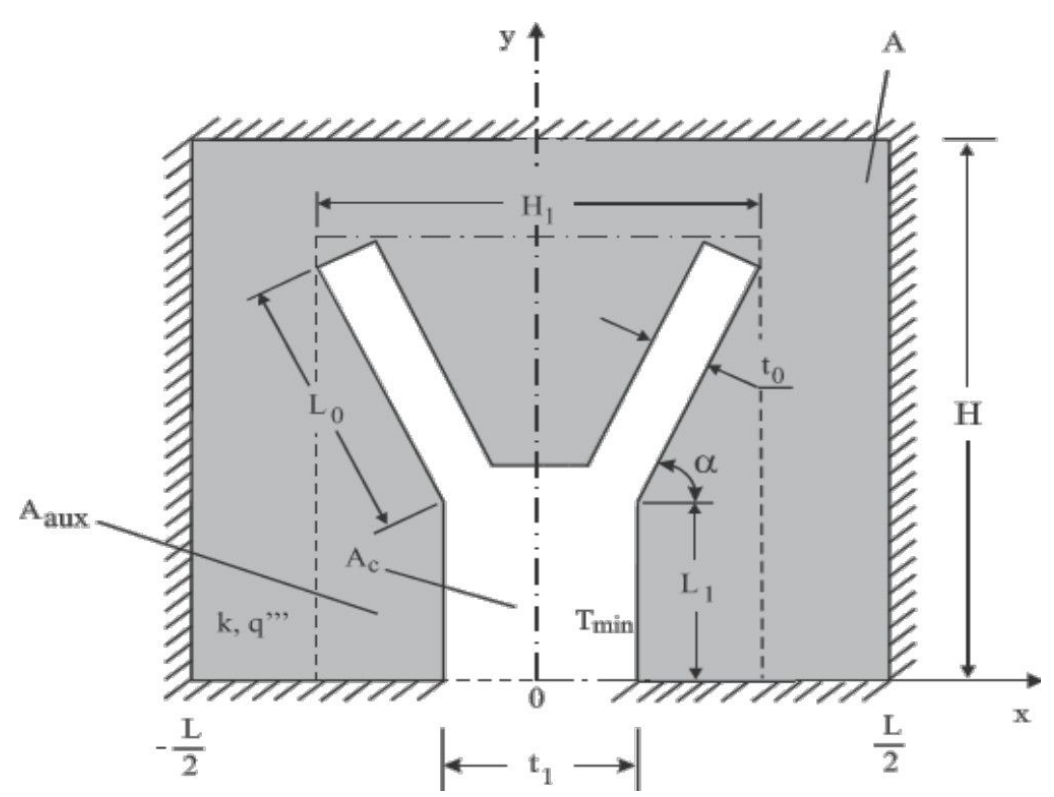

Figure 1: Computational Domain of Y-shaped cavity into a solid with internal heat generation

More details of mathematical model can see in [17]. The main objective is to minimize the dimensionless maximum excess of temperature $\left(\theta_{\max }\right)$ in the solid domain, given by dimensionless equation:

$$
\theta_{\max }=\frac{T_{\max }-T_{\min }}{q^{\prime \prime \prime} A / k}
$$

\section{NUMERICAL MODEL}

The function represented by Equation (1) is determined numerically by solving the temperature field in every assumed configurations $\left(H / L, t_{1} / t_{0}, L_{1} / L_{0}, \alpha\right)$ and calculating $\theta_{\max }$ to see whether $\theta_{\max }$ can be minimized by varying the configuration. The numerical solution is performed with the Finite Element Method (FEM), see Refs. [24, 25]. The domain is discretized with linear triangular elements. The solution is performed in MATLAB environment, precisely the PDE (partial-differential-equations) toolbox [26]. The grid was non-uniform in both $x$ and $y$ directions, and varied from one geometry to the next. The appropriate mesh size was determined by successive refinements (h-adaptively), increasing the number of elements four times from the current mesh size to the next mesh size, until the following criterion is satisfied:

$$
\left|\left(\theta_{\max }^{i}-\theta_{\max }^{i+1}\right) / \theta_{\max }^{i}\right|<1 \times 10^{-4} \mid
$$

where $\theta_{\mathrm{i}}$ represents the maximum value of the temperature evaluated with current mesh, $\theta_{i+1}$ represents the temperature evaluated with next refined mesh (which has four elements more than the previous mesh).

Table 1 illustrates the refinement mesh process with the aim to find an independent mesh that does not influence results of temperature field. The following results were performed by using a range between 2240 and 143360 triangular elements. The independent mesh is achieved for the third refinement (35840 elements). The grid independence test is performed for a fixed geometry with the following parameters: $\phi=0.05, \psi=0.5, H / L=1.0, t_{1} / t_{0}=2.0, L_{1} / L_{0}=0.5, \alpha=$ 0.94 . 
Table 1: Grid independence test

\begin{tabular}{ccc}
\hline Number of Elements & $\boldsymbol{\theta}_{\max }$ & $\left|\left(\boldsymbol{\theta}_{\max }^{\mathrm{i}}-\boldsymbol{\theta}^{\mathrm{i}+1}{ }_{\max }\right) / \boldsymbol{\theta}_{\max }^{\mathrm{i}}\right|$ \\
\hline $\mathbf{2 2 4 0}$ & 0.079046 & $5.6600 \times 10^{-4}$ \\
$\mathbf{8 9 6 0}$ & 0.079001 & $1.8978 \times 10^{-4}$ \\
$\mathbf{3 5 8 4 0} *$ & 0.078986 & $5.6971 \times 10^{-5}$ \\
$\mathbf{1 4 3 3 6 0}$ & 0.078982 & ----------- \\
\hline \multicolumn{2}{c}{$*$ Independent mesh } \\
\hline
\end{tabular}

The results obtained in grid independence test were compared to previous results found in Refs.[18] and [21]. The comparison is depicted in Table 2.

Table 2: Comparison between the results achieved with the present model and those showed in literature.

\begin{tabular}{cc}
\hline Reference & $\boldsymbol{\theta}_{\max }$ \\
\hline Present Work & 0.0762 \\
Lorenzini et al. [18] & 0.0762 \\
Lorenzini et al. [21] & 0.0762 \\
\hline
\end{tabular}

\section{SIMULATED ANNEALING}

The combinatorial optimization algorithm Simulated Annealing (SA) was proposed firstly in Kirkpatrick et al. [1] and it has a heuristic based in production of resistant materials, like glass or metal, submitted to annealing process. Initially the material is heated at high level temperatures and then cooled slowly to obtain the minimization of energy between the atoms of the material. The algorithm of Kirkpatrick et al. [1] applies the optimization proposed by Metropolis et al. [2] for various temperature levels.

Based on the heuristic presented, the algorithm SA starts the search for the possible solutions in the search space and accept new solutions according with a probability given by Eq. (3), which depends on current iteration temperature of algorithm. According to Eglese [3], the initial temperature, the method as the temperature decrease, the number of neighbor proposes at each level of temperature, the reannealing parameter and the stop criterion, are named cooling schedule. For high algorithm temperatures of the annealing process the algorithm proposes distant new geometric neighbor point and has more probability to accept bad solutions, i.e., distant from the optimal shape. As the algorithm temperature decreases the new proposed neighbor points are near from the current point and the probability to accept bad solutions decreases considerably.

$$
P=\frac{1}{1+\exp \left(\frac{\Delta}{\max (T)}\right)}
$$

In Eq. (3) $\Delta$ is the difference between the value returned by Objective Function of the new configuration in relation to Objective Function value for the best evaluated point. More precisely, when a new geometry neighbor is proposed in comparison with the current solution, the value of Objective Function, which it is intended to be minimized, is calculated for this new geometry (neighbor). Afterwards, it is calculated $\Delta$ subtracting the value of Objective Function for the neighbor solution and Objective Function for the current solution. The acceptance probability of solution also depends on the actual algorithm iteration. Once the numerical model used here led to an achievement of different temperatures for each degree of freedom, for calculation with Eq. (3) it is used the highest temperature of domain [26].

To apply the SA algorithm in the geometric optimization of the Y-shaped cavity, it was needed the same discretization of the space search realized in the study of Lorenzini et al. [21]. The implementation of SA was run in MATLAB environment. More precisely, it is used the Global Optimization Toolbox with a specific function to SA execution named simulannealbnd. 
The SA solver of the MATLAB is extremely adaptable, i.e., it is possible to develop new algorithms for several functions as cooling schedule, annealing function or the acceptance function.

The present work applied the SA algorithm for the same problem previously studied in Refs. $[16,21]$ with the Exhaustive Search (ES) and Genetic Algorithm (GA), which were employed to seek for the optimal shapes. In the present study, the main intention is to find the best configurations for SA (cooling schedule) that combined with Constructal Design can be employed to seek for the best geometries of complex cavity shapes. Twenty samples with 30 executions of the SA is realized trying to found the once optimized ratio of $L_{1} / L_{0},\left(L_{1} / L_{0}\right)_{0}$, and the twice optimized angle $\alpha, \alpha_{00}$, for three different values of the ratio $t_{1} / t_{0}: t_{1} / t_{0}=10.0,11.0$ and 12.0, which represents the optimal region for the ratio $t_{1} / t_{0}$ and with the fixed parameters studied here, see Lorenzini et al. [21]. In this analysis it was extracted the mean percentage of achievement of the global optimal geometries with each cooling schedule.

For optimization of two DOF of the problem, the search space formed by $L_{1} / L_{0}$ and $\alpha$ is discretized in the same solution space employed for GA algorithm used in Lorenzini et al. [21]. The SA is evaluated for 15 different values of DOF $L_{1} / L_{0}$, as can be seen in the vector represented in Eq. (4), while the DOF $\alpha$ is varied in the interval $1.00 \leq \alpha \leq 1.57$ with a step of $\Delta \alpha=0.01$. The results present in Lorenzini et al. [21] were used to validate the optimal configuration found here with SA and it is also used as stop criterion for the algorithm.

$$
L_{1} / L_{0}=\left|\begin{array}{c}
0.001 \\
0.002 \\
0.003 \\
0.005 \\
0.007 \\
0.01 \\
0.02 \\
0.03 \\
0.05 \\
0.07 \\
0.1 \\
0.3 \\
0.5 \\
0.7 \\
1
\end{array}\right|
$$

The SA algorithm requires a function that must propose the neighbor solutions from the current point during the optimization process, this function is called annealing function, which is developed in the present study for optimization with two DOFs $\left(L_{l} / L_{0}, \alpha\right)$. The proposal of new neighbor for the possible values of DOFs must respect the discretized space, i.e., generating in a random way the values inside the search space. The random number function used is the default function of MATLAB together with an adaptation calculus for generation of valid values in search space and inside of the discretization established for each degree of freedom.

The cooling schedules observed in this work has the same initial parameters of SA, defined in the start of algorithm, only difference is the temperature decrease that is the investigation goal. Six functions of cooling was studied, the default functions of MATLAB Optimization Toolbox (Exponential, Fast and Boltz) and more three hybrid and customized function which will be called here: BoltzExp, ConstExp1 and ConstExp2. These functions are respectively represented by the following equations:

$$
T_{i}=T_{0} \times 0.95^{k_{s a}}
$$




$$
\begin{aligned}
& T_{i}=\frac{T_{0}}{k_{s a}} \\
& T_{i}=\frac{T_{0}}{\ln \left(k_{s a}\right)} \\
& T_{i}=\left\{\begin{array}{cc}
\frac{T_{0}}{\ln \left(k_{s a}\right)} & : k_{s a} \leq 80 \text { for } T_{0}=100 \\
T_{0} \times 0.95^{\left(k_{s a}-T_{0} / 2\right)} & : k_{s a}>80 \text { for } T_{0}=100
\end{array}\right. \\
& T_{i}=\left\{\begin{array}{lc}
T_{0} & : k_{s a} \leq \frac{T_{0}}{2} \\
T_{0} \times 0.95^{\left(k_{s a}-T_{0} / 2\right)} & : k_{s a}>\frac{T_{0}}{2} \\
T_{0} & \leq \frac{T_{0}}{2} \\
T_{0} \times 0.9^{\left(k_{s a}-T_{0} / 2\right)} & : k_{s a}>\frac{T_{0}}{2}
\end{array}\right.
\end{aligned}
$$

where $k_{\mathrm{sa}}$ is the annealing parameter, which is the same value for iteration number until reannealing, i.e., the number of iterations before the recalculation of temperature. Reannealing raises the temperature after the algorithm accepts a certain number of new points, and start the search again at the higher temperature. The number of iterations after reannealing is configured in MATLAB Optimization Toolbox, which by default is considered 100. $T_{\mathrm{i}}$ in above equations represents the temperature in the current iteration and $T_{0}$ represents the initial temperature of the algorithm, that is assumed $T_{0}=100$ by default.

In this work the proposed cooling temperature, named Boltzexp, is a hybrid form between the models described in Equations (5) and (6). Basically, the function consists on the evaluation of the temperature obtained with the two equations and the employment of the lowest temperature obtained with both methods in the current iteration. The value $k_{\mathrm{sa}}=80$ in Eq. (8), represents the number of iteration when the function changes the model of decrease temperature, i.e., from Boltz to Exponential.

Two more cooling schemes are proposed, the ConstExp1 and ConstExp2, represented by Eqs. (9) and (10), respectively. These models are very similar, the only difference between them is the constant of decrease in the cooling temperature, which are respectively given by 0.95 and 0.9. Both schedules maintain the temperature fixed in the initial steps until the number of iterations is equal to the half of initial temperature $\left(T_{0}\right)$. In first steps of those models, the algorithm runs freely at initial temperature and with full probability to accept new points with the purpose to find a better initial point until the temperature decrease. In Figure 2 it is possible to observe the behavior of temperature decay with the number of iterations for the several cooling schedules evaluated here. 


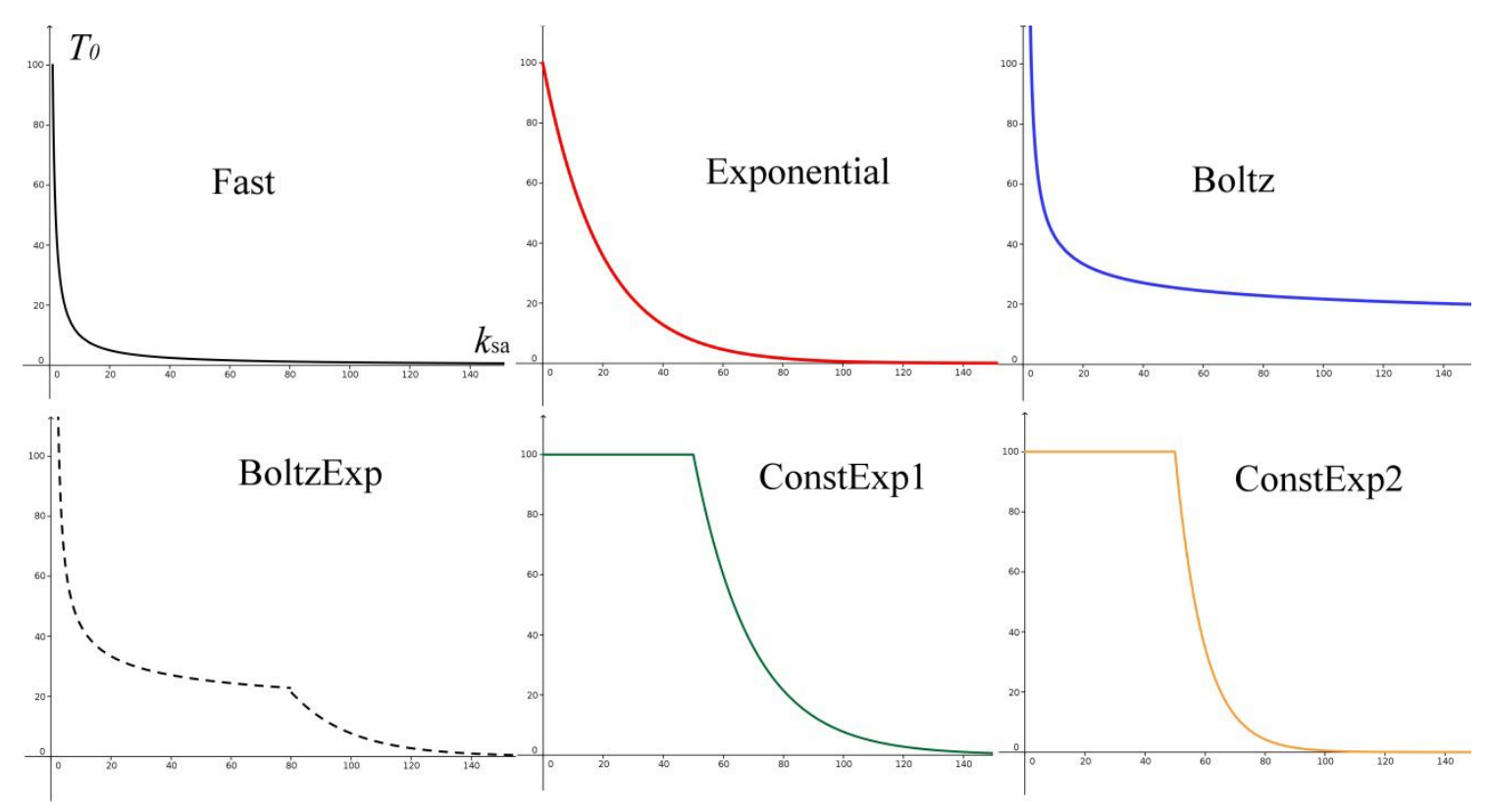

Figure 2: The behaviour of algorithm temperature as a function of the number of iterations for the six different cooling schedule of $S A\left(T_{0}=100\right.$ for all models $)$.

\section{RESULTS AND DISCUSSION}

In this study, it is evaluated only the SA parameter named decrease of temperature. The other parameters are maintained fixed with the sole interest to evaluate the influence of cooling schedule function in the search for the optimal shapes of the studied Y-shaped cavity.

As above mentioned, the study focused in the optimal region for $t_{1} / t_{0}$ reported in Lorenzini et al. [21]. For all geometries evaluated, the parameter $H / L$ is considered constant $H / L=1.0$ and the areas fractions are $\phi=0.05$ and $\psi=0.3$. The results of optimal configuration in Lorenzini et al. [21] were used as stop criterion for the algorithm SA.

Table 3 shows for each cooling schedule the percentage of achievement of the optimal geometry, i.e., which minimizes the dimensionless maximum excess of temperature $\left(\theta_{\max }\right)$. From the 30 executions for each sample (in a total of 20 samples) for each cooling schedule and for each evaluated ratio of $t_{1} / t_{0}$, it was possible to reach to statistics measures of the achievement of global optimal geometry. Results of Table 3 reveal that SA algorithm is not deterministic and they show the importance of statistical analysis to understand the behavior and influence of the different parameters in optimization algorithms. For example, Fast cooling schedule reaches to the optimal shape only $12.94 \%$ of the evaluated samples. On opposite, the ConstExp2 leads to an achievement of $62.44 \%$, in average, of the optimal shapes, showing that the new proposed cooling schedules are more effective in the search for the global optimal shapes than the default models presented in MATLAB Optimization Toolbox. Among the evaluated cooling schedules, the worst performance was obtained with the Fast method, which clearly is not recommended for this kind of problem. Even for a specific sample, where the Fast cooling schedule reaches to the optimal in $36.67 \%$ of the simulations, the performance was inferior to the average performance of other functions as Boltz, BoltzExp, ConstExp1 and ConstExp2. It can also be noticed that the method Exponential also has a lower percentage to find the global optimal shape, the mean percentage is only $27.50 \%$ with a standard deviation of $16.86 \%$. The hybrid cooling schedules allowed the increase of the number of times where the global optimal geometry for Y-shaped cavity with two DOFs is find, being in this primary analysis more reliable for combination with Constructal Design for optimization of complex cavities in heat transfer realm. However, these methods (BoltzExp, ConstExp1 and ConstExp2) also increase the magnitude of standard deviation, which can be negative for representation of local effects of degrees of freedom over the thermal resistance of the problem. The ideal case is that one which 
in average reaches to a high number of cases where the geometry is optimized and with a few variations (standard deviation) when the geometrical parameters are varied.

Table 3: Percentage distribution of statistics in the achievement of optimal shapes for each cooling schedule.

\begin{tabular}{ccccccc}
\hline $\begin{array}{c}\text { Statistical Measures / } \\
\text { Function }\end{array}$ & Fast & Exponential & Boltz & BoltzExp & ConstExp1 & ConstExp2 \\
\hline Mean & 12.94 & 27.50 & 42.72 & 54.39 & 54.67 & 62.44 \\
\hline Absolute Deviation & 8.50 & 14.75 & 14.71 & 17.98 & 19.80 & 22.68 \\
\hline Standard Deviation & 10.11 & 16.86 & 17.09 & 20.93 & 23.57 & 25.63 \\
\hline Variance & 102.10 & 284.23 & 292.10 & 438.23 & 555.63 & 656.82 \\
\hline Mode & 3.33 & 6.667 & 33.33 & 30.00 & 20.00 & 83.33 \\
\hline Median & 11.67 & 30.00 & 36.66 & 53.33 & 56.67 & 71.67 \\
\hline Smaller & 0.00 & 0.00 & 13.33 & 16.67 & 10.00 & 10.00 \\
\hline Largest & 36.67 & 60.00 & 73.33 & 90.00 & 93.33 & 96.67 \\
\hline
\end{tabular}

The Table 4, ANOVA was used for proof the difference between the cooling schedules analyzed. In this analysis was employed the one-way ANOVA, because the percentage sample for each treatment, or cooling schedule, are independents [27]. Also was observed twenty rounds of thirty runs of the SA for each value of $t_{1} / t_{0}(10,11$ and 12) in optimal region for this case. So, were evaluated six different treatments, each one of them with sixty samples of the percentage in find the optimal shapes at thirty optimization processes. Therefore the model employed for ANOVA is balanced, i.e., the same number of samples for each treatment. The PValue, shown in Table 4, is very minor than the significance level 0.001 , this indicates that at least one distribution of the percentages is different [27].

Table 4: Analysis of variance between all cooling schedules.

\begin{tabular}{cccccc}
\hline $\begin{array}{c}\text { Source of } \\
\text { Variation }\end{array}$ & $\begin{array}{c}\text { Degrees of } \\
\text { Freedom }\end{array}$ & $\begin{array}{c}\text { Sum of } \\
\text { Square }\end{array}$ & Mean Squares & F-Value & P-Value \\
\hline Schedules & 5 & 107143 & 21428.6 & 55.2 & $2.56401 \mathrm{e}-42$ \\
\hline Residuals & 354 & 137417.1 & 388.2 & --- & -- \\
\hline Total & 359 & 244560 & --- & --- & --- \\
\hline
\end{tabular}

Figure 3 depicts the percentage of global optimization for each cooling schedule. It can be seen a similarity between three last cooling schedules: BoltzExp, ConstExp1 and ConstExp2. In average these cooling schedules are better than the other three studied methods (Fast, Exponential and Boltz). Results also showed that in local conditions (one specific sample) the methods ConstExp1 and ConstExp2 reach to the global optimal shape only in $10 \%$ of the cases and for other specific samples they reached to magnitudes of achievement higher than $90 \%$, which can be seen in black traces. It is also important to mention that for these cooling schedules local optimal shapes with a performance similar to that obtained for the global optimal shapes were reached. In this sense, in general, the hybrid cooling schedules showed for the present study the most recommended functions for application of SA and Constructal Design for geometrical optimization of heat transfer problems. 


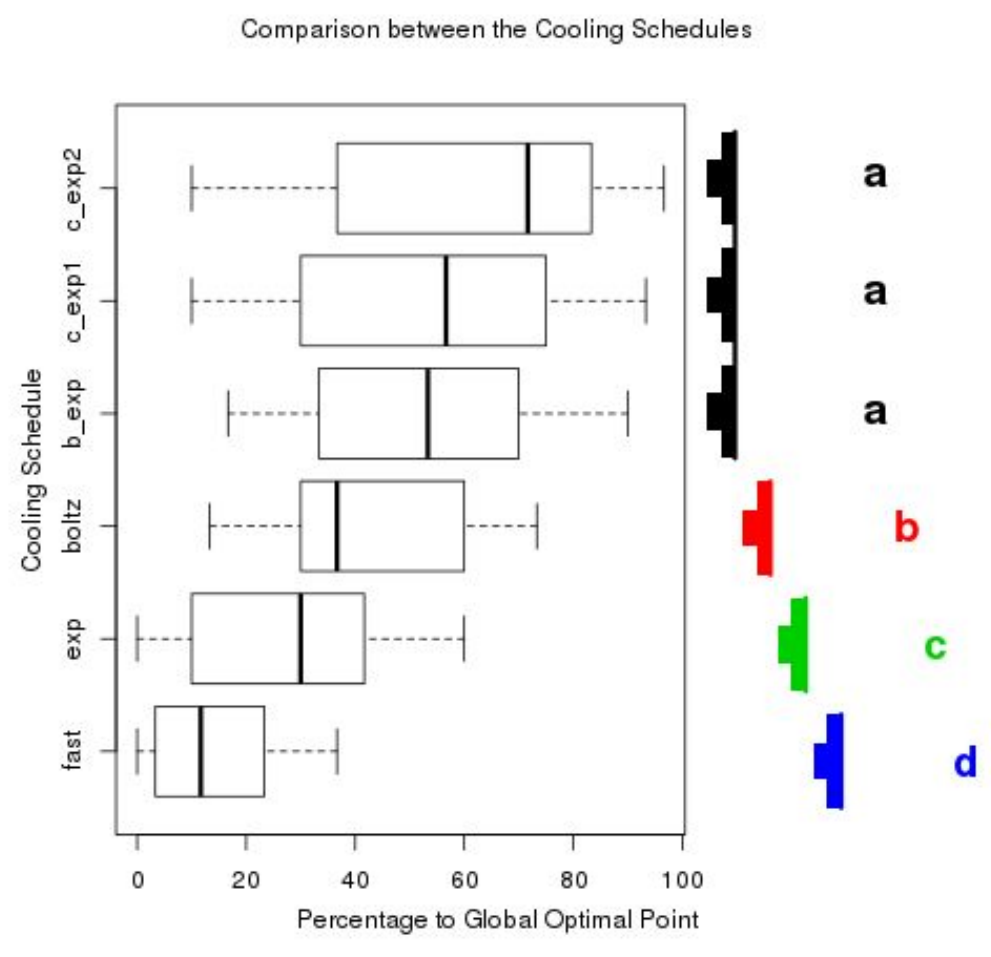

Figure 3: The comparison and classification between analysed cooling schedules (b_exp= BoltzExp;c_exp1 $=$ CosntExp1;c_exp2 $=$ ConstExp2).

To corroborate the previous findings, Figure 3 also shows the classification of all studied cooling schedules is performed based on pairwise comparison and the mean value of percentage distribution of achievement of global optimal shape. Figure 3 was made with data generated by Software-R, a free software environment for statistical computing. Moreover, the Tukey test was employed for generated this graph. The level of significance employed in the Tukey test was 0.05 , what resulted in a confidence rate of $95 \%$. Results clearly revealed the inferiority of Fast and Exponential cooling schedules and the superiority of new schedules to find the optimal geometries in the present studied cases.

\section{CONCLUSION}

In this work, six functions of the cooling schedule were compared and classified. This analysis employed the SA algorithm combined with Constructal Design for geometrical optimization of complex Y-shaped cavities. Results showed that SA is a very useful tool for optimization in the framework of Constructal Design, but this meta-heuristic requires an adequate selection of its main parameters, mainly the cooling schedule. For the case with more degrees of freedom when ES is not applicable, the reliable of meta-heuristics used is very important for geometric optimization, as well as, the evaluation of effects of each degree of freedom over the thermal performance of the system.

In the present study it was noticed that the Fast cooling schedule led to the worst performance, reporting several local optimal geometries for the Y-shaped cavity intruded into conductive solid wall with internal heat generation. Even when the best results obtained with Fast cooling schedule are taken into account, the performance is inferior to those reached with hybrid cooling schedules (BoltzExp, ConstExp1 and ConstExp2). The other two functions for cooling schedule (Exponential and Boltz) led to intermediate performance.

The best results were obtained with the new cooling schedules proposed (BoltzExp, ConstExp1 and ConstExp2). The increase in the achievement of global optimal shapes for the 
hybrid cooling schedules are concerned with the increase of freedom given by the algorithm to explore the search space in the initial iterations. When the algorithm starts with bad initial point, he has more iteration to change to a better point until the beginning of temperature decay. It is important to mention that the new cooling schedules were the only functions that reach to a maximum percentage of more than $90 \%$ in the achievement of optimal shapes.

In the future, the results generated in this paper will be employed in a same class of problem but with a new geometry, the double-T shaped cavity. This geometry has a more degrees of freedom and you complete optimization would consume a large computational effort with Exhaustive Search and will need the employment of SA for geometric optimization.

\section{AGRADECIMENTOS}

Professors L. A. Isoldi, L. A. O. Rocha and E. D. dos Santos thank CNPq for research grant.

\section{REFERENCES}

1. Kirkpatrick S, Gelatt CD, Vecchi MP. Optimization by Simulated Annealing. Science. New Series. 1983; 220 (4598): 671-680.

2. Metropolis N, Rosenbluth AW, Rosenbluth MN, Teller AH. Equation of State Calculations by Fast Computing Machines. J. Chem. Phys. 1953; 21: 1088-1092.

3. Eglese RW. Simulated Annealing: A tool for Operational Research. Eur. J. Oper. Res. 1990; 46:.271281.

4. Bertsimas D, Tsitsiklis J. Simulated Annealing, Statistical Science 1993; 8 (1): 10-15.

5. Szu H. Hartley R. Fast Simulated Annealing, Phys. Lett. A 1987; 122: 157-162.

6. Hajek B. Cooling Schedules for Optimal Annealing. Math. Oper. Res. 1988; 13 (2): 111-129.

7. Ingber L.Very Fast Simulated Re-annealing, Math. Comput. Model. 1989; 12 (8): 967-973.

8. Ingber L. Simulated Annealing: Patrice versus Theory, Math. Comput. Model. 1993; 18 (11): 29-57.

9. Nourani Y, Andresen B. A comparison of simulated annealing cooling strategies, J. Phys. A: Math. Gen. 1998; 31: 8373-8385.

10. El-Bouri A, Azizi N, Zolfaghari S. A comparative study of a new heuristic based on adaptive memory programming and simulated annealing: The case of job shop scheduling, Eur. J. Oper. Res. 2007; 177: 1894-1910, doi: 10.1016/j.ejor.2005.12.013.

11. Schneider JJ, Puchta M. Investigation of acceptance simulated annealing - A simplified approach to adaptive cooling schedules, Physica A 2010; 389 (24): 5822-5831, 10.1016/j.physa.2010.08.045.

12. Kastanya D. Evaluating the effects of cooling schedule on the performance of ADORE, Ann. Nucl. Energy 2013; 62: 483-486, doi: 10.1016/j.anucene.2013.06.019.

13. Brusco MJ. A comparison of simulated annealing algorithms for variable selection in principal component analysis and discriminant analysis, Comput. Stat. Data An. 2014; 77: 38-53, doi: 10.1016/j.csda.2014.03.001.

14. Bejan A. Shape and Structure, from Engineering to Nature. Cambridge University Press, Cambridge, UK; 2000.

15. Biserni C, Rocha LAO, Bejan A. Inverted Fins: Geometric Optimization of the Intrusion into a Conducting Wall, Int. J. Heat Mass Tran. 2004; 47: 2577-2586.

16. Estrada E, Dos Santos ED, Isoldi LA, Rocha LAO. Genetic Algorithm and Constructal Design for Geometric Optimization of Y-Shaped. In: V MCSUL, Rio Grande - RS. V Conferência Sul de Modelagem Computacional, 2012. v. 1. p. 45-50.

17. Lorenzini G, Biserni C, Isoldi LA, Dos Santos ED, Rocha LAO. Constructal Design Applied to the Geometric Optimization of Y-shaped Cavities Embedded in a Conducting Medium. J. Electronic Packaging. 2011; 133: 041008-1 - 041008-8, doi: 10.1115/1.4005296.

18. Lorenzini G, Garcia FL, Dos Santos ED, Biserni C, Rocha LAO. Constructal design applied to the optimization of complex geometries: T-Y-shaped cavities with two additional lateral intrusions cooled by convection, Int. J. Heat Mass Tran. 2012; 55 (5 - 6): 1505-1512, doi: 10.1016/j.ijheatmasstransfer.2011.10.057.

19. Lorenzini G, Biserni C, Link FB, Isoldi LA, Dos Santos ED, Rocha LAO. Constructal Design of TShaped Cavity for Several Convective Fluxes Imposed at the Cavity Surfaces. J. Eng. Thermophys. 2013; 22 (4): 309-321, doi: 10.1134/S1810232813040048. 
20. Hajmohammadi MR, Poozesh S, Campo A, Nourazar SS. Valuable Reconsideration in the Constructal Design of Cavities. Energy Convers. Manage. 2013; 66: 33-40, doi: 10.1016/j.enconman.2012.09.031.

21. Lorenzini G, Biserni C, Estrada ESD, Dos Santos ED, Isoldi LA, Rocha LAO. Genetic Algorithm Applied to Geometric Optimization of Isothermal Y-Shaped Cavities. J. Electronic Packaging. 2014; 136 (3): 031011-1-031011-8, doi: 10.1115/1.4027421.

22. Lorenzini G, Biserni C, Estrada ED, Isoldi LA, Dos Santos ED, Rocha LA.O. Constructal Design of Convective Y-Shaped Cavities by Means of Genetic Algorithm. J. Heat Trans-T ASME. 2014; 136 (7): 071702-071702-10, doi: 10.1115/1.4027195.

23. Xie Z, Chen L, Sun F. Geometry Optimization of T-Shaped Cavities According to Constructal Theory. Math. Comput. Model. 2010; 52 (9-10):1538-1546, doi: 10.1016/j.mcm.2010.06.017.

24. Reddy JN, Gartling DK. The Finite Element Method in Heat Transfer and Fluid Dynamics. CRC, Boca Raton, Florida,USA;1994.

25. Zienkiewicz OC, Taylor RL. The finite element method: The Basis. Volume 1, ButterworthHeinemann, Oxford,.Ed 5; 2000.

26. MATLAB User's Guide, Version 6.0.088, Release 12, The Mathworks Inc., Natick, MA, 2000.

27. Hines WW, Montgomery DC, Goldsman DM, Borror CM. ,2006. Tradução: VR Lima, Probabilidade e Estatística na Engenharia. Cap.12, Rio de Janeiro, LTC. 